\title{
Physiological starvation increases EGF-Ras-MAPK pathway activity during $C$. elegans vulval induction
}

\author{
Stéphanie Grimbert ${ }^{1}$, Amhed Missael Vargas Velazquez ${ }^{2} \&$ Christian Braendle $^{1^{*}}$ \\ ${ }^{1}$ Université Côte d'Azur, CNRS, Inserm, IBV, Nice, France, 06100 Nice, France \\ ${ }^{2}$ Institute de Biologie de l'Ecole Normale Supérieure, CNRS UMR 8197 and Inserm U1024, \\ Paris, France
}

*Correspondence: braendle@unice.fr

Running Title: Starvation modulation of C. elegans vulval induction

Key Words: Caenorhabditis, LIN-3, Mutational penetrance, TOR-S6K, Vulval cell fate patterning 


\begin{abstract}
Studying how molecular pathways respond to ecologically relevant environmental variation is fundamental to understand organismal development and its evolution. Here we characterize how starvation modulates Caenorhabditis elegans vulval cell fate patterning - an environmentally sensitive process, with a nevertheless robust output. Past research has shown many vulval mutants affecting EGF-Ras-MAPK, Delta-Notch and Wnt pathways to be suppressed by environmental factors, such as starvation. Here we aimed to resolve previous, seemingly contradictory, observations on how starvation modulates levels of vulval induction. Using the strong starvation suppression of the Vulvaless phenotype of lin-3/egf reduction-of-function mutations as an experimental paradigm, we first tested for a possible involvement of the sensory system in relaying starvation signals to affect vulval induction: mutation of various sensory inputs, DAF2/Insulin or DAF-7/TGF- $\beta$ signaling did not abolish lin-3(rf) starvation suppression. In contrast, nutrient deprivation induced by mutation of the intestinal peptide transporter gene pept-1 or the TOR pathway component rsks-1 (the orthologue of mammalian P70S6K) very strongly suppressed lin-3(rf) mutant phenotypes. Therefore, physiologically starved animals induced by these mutations tightly recapitulated the effects of external starvation on vulval induction. While both starvation and pept-1 RNAi were sufficient to increase Ras and Notch pathway activities in vulval cells, the highly penetrant Vulvaless phenotype of a tissue-specific null allele of lin-3 was not suppressed by either condition. This and additional results indicate that partial lin-3 expression is required for starvation to affect vulval induction. These results suggest a cross-talk between nutrient deprivation, TOR-S6K and EGF-Ras-MAPK signaling during C. elegans vulval induction.
\end{abstract}

\title{
Introduction
}

Organismal development is inherently sensitive to environmental variation, and specific environmental conditions, such as nutrient availability or temperature, may also reflect instructive cues controlling growth and other critical developmental decisions, such as developmental timing, diapause entry and production of alternative phenotypes. Developmental integration of environmental cues has become increasingly understood, primarily in the context of nutritional and metabolic regulation of developmental decision, growth and lifespan, such as in Drosophila and C. elegans (Flatt et al. 2013). In contrast to the understanding of such instructive environmental cues in development, very little is known about how environmental variation impacts other, highly diversified developmental processes, seemingly unaffected by the environment as they maintain their function and corresponding phenotypic outputs when facing such variation. However, such stability of the phenotypic output may go in hand with an underlying flexibility of developmental mechanisms in changing environmental (and genetic) conditions (Félix and Barkoulas 2015; True and Haag 2001; Paaby and Rockman 2014).

C. elegans vulval development provides one clear example of a developmental process, which robustly generates an invariant phenotypic output (cell fate pattern) although activities and interactions of underlying molecular signaling pathways are environmentally sensitive (Braendle and Félix 2008; Grimbert and Braendle 2014). $C$. elegans vulval cell fate patterning involves a network of conserved signaling pathways, EGF-Ras-MAPK, Delta-Notch and Wnt pathways that reliably establish a stereotypical cell fate pattern of hypodermal vulval precursor cells (VPCs) (Félix 2012; Sternberg 2005) (Figures 1A and 1B). The VPCs represent a subset of Pn.p cells, P3.p to P8.p, 
competent to adopt vulval cell fates. Competence of vulval precursor cells is established during L1-L2 stages through expression of the Hox gene lin-39 and Wnt signals from the posterior (Penigault and Félix 2011; Salser et al. 1993). The key events of the vulval patterning process take place from mid L2 to early L3 stage and involve intercellular signaling between the gonadal anchor cell (AC) and the VPCs (Figures 1A and 1B). In brief, LIN-3/EGF ligand released from the AC induces the primary $\left(1^{\circ}\right)$ vulval cell fate by activating the EGF-Ras-MAPK pathway in P6.p, which receives the highest dose of this signal (Hill and Sternberg 1992; Grimbert et al. 2016). EGF-Ras-MAPK activation induces production of a lateral signal via the Delta-Notch pathway, promoting $2^{\circ}$ and inhibiting $1^{\circ}$ cell fate in the neighboring cells, P5.p and P7.p (Greenwald et al. 1983; Sternberg and Horvitz 1986; Berset et al. 2001; Yoo et al. 2004). Moreover, a switch from the canonical LET-60/Ras-LIN-45/Raf pathway to a LET-60/Ras-RGL-1-RAL1/RAL signaling pathway can promote the $2^{\circ}$ cell fate in P5.p and P7.p (Zand et al. 2011). The remaining three VPCs, although competent, adopt non-vulval cell fates $\left(3^{\circ}\right.$ for P4.p and P8.p, and $3^{\circ}$ or $4^{\circ}$ for P3.p) as they do not receive sufficient doses of either EGF-Ras-MAPK or Delta-Notch signal. In addition to EGF-Ras-MAPK and DeltaNotch pathways, the canonical Wnt signaling pathway may also be involved in vulval induction: overactivation of the Wnt pathway, e.g. through pry-1/Axin mutation, increases vulval induction of diverse hypoinduced mutants (Braendle and Félix 2008; Gleason et al. 2002; Seetharaman et al. 2010). C. elegans vulval development thus involves a regulatory network of three key molecular cascades and their cross-talk contributes to a reliable and precise patterning output in the presence of both genetic and environmental perturbations (Braendle et al. 2010; Braendle and Félix 2008; Félix and Barkoulas 2012; Gleason et al. 2002; Hoyos et al. 2011; Milloz et al. 2008).

Despite the apparent robustness of $C$. elegans vulval cell fate patterning to environmental perturbations, multiple reports demonstrate that this process is nevertheless responsive to environmental and physiological inputs (Braendle and Felix 2009; Braendle et al. 2008; Félix 2012; Félix and Barkoulas 2012; Sternberg 2005) (summarized in Figure 1C). In early mutagenesis screens, various Vulvaless mutations (lin-2, lin-3, lin-7, lin-24, lin-33 and let-23) were found to be suppressed by starvation and/or dauer passage (Ferguson and Horvitz 1985), suggesting that vulval inductive pathways, such as EGF-Ras-MAPK, are environmentally sensitive. Detailed studies later confirmed that specific chemical elements, such as zinc (Bruinsma et al. 2002; Yoder et al. 2004), and growth conditions indeed modulate vulval inductive signaling. Moghal et al. (2003) reported that liquid culture increases vulval inductive signals via the neuronally expressed heterotrimeric Gaq protein, EGL-30. The EGL-30/Gaq signal is transduced via the voltage-gated calcium channel, EGL-19, in muscle cells and its positive effect on vulval induction is mediated by Wnt signaling via BAR-1/ $\beta$ Catenin (Moghal et al. 2003). Battu et al. (2003) further found evidence for a starvation signal that negatively affects vulval induction via the sensory system. In this study, starvation conditions were found to suppress vulval hyperinduction, for example, induced by let-60/Ras $(g f)$. This negative starvation effect requires the G-proteincoupled receptor SRA-13 to modulate EGF-Ras-MAPK activity (Battu et al. 2003). Consistent with a sensory-mediated nutritional modulation of vulval signaling, compromised DAF-2/Insulin signaling recapitulates the results that starvation negatively affects vulval induction (Battu et al. 2003; Nakdimon et al. 2012). In addition, another study (Nakdimon et al. 2012) indicates that DAF-18/PTEN negatively regulates EGF-Ras-MAPK activity during vulval induction, further supporting the view that multiple key metabolic and sensory pathways interact with vulval signaling pathways. 
Braendle \& Félix (2008) examined the effects of diverse environmental conditions (different temperatures, starvation, liquid culture, dauer passage) on vulval induction using a diverse set of known mutations in EGF-Ras-MAPK, Delta-Notch and Wnt pathways. A majority of mutations showed variable penetrance depending on the environment, with starvation and dauer environments showing the most pronounced effects (Braendle and Félix 2008). Consistent with the results of Ferguson \& Horvitz (1985), yet seemingly contrary to the results obtained by Battu et al (2003), starvation increased vulval inductive levels, leading to drastic suppression of the Vulvaless phenotypes caused by lin-3(rf) and let-23/egfr(rf) mutations (Braendle and Félix 2008). Genetic analysis suggests that this starvation signal acts at the level or upstream of LET-23/EGFR (Braendle and Félix 2008). The positive effect of starvation on vulval induction was further confirmed by quantification of pathway activities in starved versus fed wild type animals: starved animals showed a significantly higher EGF-RasMAPK pathway activity in the $1^{\circ}$ cell, P6.p, whereas Delta-Notch activity was higher in $2^{\circ}$ cells, P5.p and P7.p (Braendle and Félix 2008). In addition, the same study found that starvation potentially modulates vulval induction via the Wnt pathway as vulval

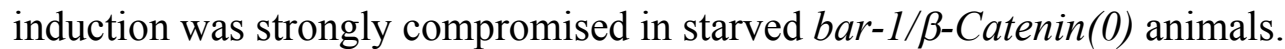

In summary, starvation experienced during larval growth may have both positive (Braendle and Félix 2008; Ferguson and Horvitz 1985) and negative (Battu et al. 2003) effects on vulval induction. While negative starvation effects have been shown to be mediated by the sensory system (Battu et al. 2003), it remains unknown how positive starvation effects are perceived and transduced to modulate vulval induction. In addition, how such, apparently antagonistically acting, starvation signals interact during vulval induction is also unknown.

Addressing these open questions, we have aimed here to quantify and characterize more precisely how starvation affects $C$. elegans vulval cell fate patterning. In particular, we ask how sensory perception of external starvation conditions versus the (internal) animal's nutrient status affect vulval induction.

\section{Material and Methods}

\section{Strains and maintenance}

Strains were maintained on NGM agar plates (55mm petri dishes, $1.7 \%$ agar) carrying a lawn of E. coli OP50 (Wood 1988; Brenner 1974). Animals were grown at $20^{\circ} \mathrm{C}$ unless indicated otherwise and both mutant and wild type strains were freshly thawed prior to experiments. Our analysis included the C. elegans N2 wild type reference strain and the mutants listed below, all of which had been previously isolated and described. LGI: egl-30(ad805), che-3(e1124)

LGII: let-23(syl), sra-13(zh13)

LGIII: daf-7(e1372), mpk-1(ku1), daf-2(e1370), lin-39(n2110), rsks-1(ok1255), zhIs4 [lip-1::GFP]

LGIV: lin-3(e1417), lin-3(n378), lin-3(mf75)

LGV: arIs92[egl-17p::NLS-CFP-lacZ, unc-4(+), ttx-3::GFP]

LGX: daf-12(rh61rh411), bar-1(mu63), bar-1(ga80), sem-5(n2019), pept-1(Ig601), osm-5(p813) 
The $\operatorname{lin}-3(m f 75)$ allele is a recently described tissue-specific transcriptionally null allele (Barkoulas et al. 2016), where two cis-regulatory elements required for anchor cell expression have been deleted using CRISPR-Cas9 (Dickinson et al., 2013).

\section{Scoring of vulval phenotypes}

The vulval phenotype was observed using Nomarski optics in early to mid L4 individuals, anaesthetized with sodium azide (Wood 1988). We counted the Pn.p progeny and determined their fates as previously described (Braendle and Félix 2008; Sternberg and Horvitz 1986).

\section{Experimental environments}

Experimental populations were age-synchronized by hypochlorite treatment and liquid arrest (27 hours) at the beginning of experiments. Individuals examined in different environments or of different strains were always scored in parallel and derived from populations kept in identical environmental conditions over at least two generations.

For starvation assays, L1 larvae were grown on standard NGM plates until they reached the mid L2 stage ( 23 hours after L1 transfer back on NGM seeded plates) unless mentioned otherwise. At this stage, animals were washed three times with sterile M9 buffer and transferred on starvation plates, i.e. unseeded NGM plates containing $1 \mathrm{mg} / \mathrm{ml}$ of ampicillin to prevent bacterial growth. After 48 hours, starved animals were transferred back to regular NGM plates seeded with E. coli OP50 and the vulval phenotype was scored when animals had reached the early or mid L4 stage (approximately 15-20 hours later). Control animals were kept on NGM plates seeded with E. coli OP50 from L1 to L4. This starvation treatment drastically reduced, yet did not completely stop, developmental progression of worms: after 48 hours most animals had developed into the early to mid-L3 individuals. Note that this starvation treatment did not induce dauer formation.

\section{RNAi experiments}

RNAi by bacterial feeding was performed as described by Timmons et al. (2001). The HT115 bacterial strain carrying the empty RNAi expression vector L4440 served as a negative control. We used RNAi plates composed of standard NGM with $50 \mathrm{ug} / \mathrm{ml}$ of ampicillin and 1mM of IPTG. Late L2/ early L3 individuals were transferred on RNAi bacteria. The vulval phenotype was scored in L4 individuals of the F1 generation. RNAi clones were selected from Ahringer's RNAi library (Kamath et al. 2003) or from Vidal's RNAi library (Rual et al. 2004). The following RNAi clones were used: pept-1 (clone K04E7.2, Ahringer Library), let-363 (clone B0261.2a, Ahringer) and rsks-1 (clone Y47D3A.16, Vidal ORFeome Library).

\section{Quantification of pept-1 RNAi effects on Ras and Notch pathway activities}

To quantify EGF-Ras-MAPK and Delta-Notch pathway activities in response to pept1 RNAi we used previously generated transgenic strains containing integrated transcriptional reporter constructs: the JU480 strain carries the egl-17::cfp-LacZ transgene (EGF-Ras-MAPK activity reporter) derived from the strain GS3582 (Yoo et al. 2004) and the AH142 strain carries the lip-1::gfp transgene (Delta-Notch activity reporter) (Berset et al. 2001). Late L2/ early L3 individuals were randomly allocated to pept-1 RNAi (K04E7.2 clone) or control (L4440 empty vector) plates. EGF-RasMAPK and Delta-Notch pathway activities were measured in lethargus L2/early L3 of the F1 generation. 
We quantified reporter gene activity in VPCs as previously described (Braendle and Félix 2008; Braendle et al. 2010) (Figure S1). In brief, CFP or GFP signal quantification was performed when individuals had reached the stage of lethargus L2/L3 or early L3. Pn.p cells of live, anesthetized individuals were first identified using DIC imaging, followed by measurement of pixel signal intensity in P5.p, P6.p and P7.p for each individual. Images were acquired using an Olympus BX61 microscope at 40X magnification, equipped with a Coolsnap HQ2 camera. To quantify signal intensity of each cell, we first selected a fixed sub-region of the same size within nuclei of target VPCs and then measured the mean signal intensity of this region. After background subtraction, we used the mean signal intensity as a measure of the corresponding signaling pathway activity in each Pn.p.

\section{Single Molecule FISH}

L3 stage animals were processed to quantify lin-3/egf mRNA levels by smFISH as described before (Barkoulas et al. 2013). In brief, they were permeabilized and hybridized with custom-made probes that bind specifically to lin-3/egf and lag-2 mRNAs, labelled with Cy5 and Alexa-594, respectively. The animals were stained with DAPI and placed in GLOX buffer for image acquisition. For each animal, we acquired Z-stacks with a Pixis 1024B camera (Princeton Instruments) mounted onto an upright Zeiss AxioImager M1 with a Lumen 200 metal arc lamp (Prior Scientific). Each Zstack was composed of 30 images spanning $20.3 \mu \mathrm{m}(0.7 \mu \mathrm{m}$ in between two acquisitions) on each filter channel. Finally, spot quantification was performed using a custom-made Matlab routine optimized for the anchor cell. Only worms with gonad length $>50 \mu \mathrm{m}$ were included in the quantifications.

\section{Statistical Analyses}

Data were transformed (e.g. Box-Cox- or log-transformed) when necessary to meet the assumptions of ANOVA procedures (homogeneity of variances and normal distributions of residuals) (Sokal and Rohlf 1981). For post hoc comparisons, Tukey's honestly significant difference (HSD) procedure was used. Statistical tests were performed using the software programs JMP v13 or SPSS v19.

\section{Data availability}

All strains generated in this study are available upon request. 


\section{Results and Discussion}

\section{Quantifying starvation effects on vulval induction in lin-3/egf(rf) mutants}

Previous studies have used different protocols to examine starvation effects on vulval induction, e.g. animals were exposed to starvation conditions for different periods of time and at different developmental time points (Battu et al. 2003; Braendle and Félix 2008; Ferguson and Horvitz 1985). We therefore first aimed to determine the starvation-sensitive periods of vulval induction using lin-3(rf) mutants known to be affected by starvation conditions (Braendle and Félix 2008; Ferguson and Horvitz 1985). Consistent with these earlier studies, a $48 \mathrm{~h}$ starvation exposure consistently increased vulval induction from late L1/early L2 to early L3 in lin-3(n378) animals (Figure 2A). Starvation sensitivity thus extends over the entire period of the vulval cell fate patterning process, with mid-L2 individuals being most sensitive. Next, we asked how the duration of starvation exposure modulates vulval induction. Exposing lin3(n378) animals to different time periods of starvation $(0-120 \mathrm{~h})$ at the mid L2 stage caused strong suppression of the Vulvaless phenotype when starved for 48 hours or more, low suppression after 19-24h of starvation, and no suppression after a brief $(2 \mathrm{~h})$ exposure to starvation or early L1 starvation (12h) in liquid medium (Figure 2B). Therefore, the extent of vulval induction depends on the duration of the starvation exposure during specific larval developmental stages.

To characterize how starvation of lin-3(rf) mutants affects vulval cell lineages, we further scored cell fate patterns in mid-L4 individuals that had been exposed to 48 hours of starvation at the mid-L2 stage. The Vulvaless phenotypes of lin-3(n378) and lin-3(e1417) were strongly suppressed by starvation (Figures $2 \mathrm{C}$ and $2 \mathrm{~F}$ ), similar to previous results (Braendle and Félix 2008). Starvation resulted in an increased mean number of induced vulval cells and a higher proportion of individuals with a canonical $2^{\circ}-1^{\circ}-2^{\circ}$ cell fate pattern (P5.p to P7.p), and these suppression effects were consistently stronger in lin-3(n378) compared to lin-3(e1417) (Figures 2C-2H). In lin-3(n378), the proportion of individuals with complete induction increased from $12 \%$ in food conditions to $50 \%$ after starvation, and most of these individuals showed a correct $2^{\circ}$ $1^{\circ}-2^{\circ}$ pattern for P5.p to P7.p (Figures $2 \mathrm{D}$ and 2E). Together, these results show that starvation can partly compensate for reduced LIN-3 signaling, confirming the previously reported positive effect of starvation on vulval induction (Braendle and Félix 2008).

\section{Compromised sensory signaling does not abolish starvation effects on vulval induction}

To understand how starvation stimuli are transduced to affect vulval induction, we first tested for an implication of the sensory system in relaying external starvation conditions. Battu et al. (2003) reported that vulval hyperinduction caused by let$60 /$ ras (gf) was significantly reduced after starvation of late L1 larvae, indicative of a negative starvation effect on vulval induction. Moreover, this study showed that mutation of the G-protein-coupled receptor sra-13 or mutations causing sensory signaling defects, such as osm-5(p813) and che-3(e1124), abolished starvation sensitivity of let-60(gf) animals, implying that observed starvation effects acted on vulval induction via components of the sensory system. We therefore tested whether disruption of the same sensory signaling components also inhibits starvation effects on vulval induction in lin-3(rf) mutants. However, we observed that starvation still significantly increased vulval induction of lin-3(n378) in sra-13(zh13), osm-5(p813) and che-3(e1124) backgrounds, comparable to the results of the lin-3(n378) single 
mutant (Table 1). Therefore, in contrast to the results of Battu et al. (2003), these sensory mutants did not abolish starvation suppression of the Vulvaless phenotype in lin-3(n378) mutants.

We next tested for an implication of TGF- $\beta$ and Insulin signaling: two key signaling pathways involved in sensory transduction of environmental stimuli, which mediate diverse developmental and metabolic responses to changes in nutritional status, e.g. during dauer formation (Fielenbach and Antebi 2008) or germline proliferation (Dalfo et al. 2012; Michaelson et al. 2010). The activity of the TGF- $\beta$ and insulin pathways are reduced upon starvation. Reducing TGF- $\beta$ pathway activity did not abolish starvation effects on vulval induction as shown by the double mutant daf7(e1372); lin-3(e1417) (Table 1). Similarly, reduced activity of the DAF-2/Insulin receptor caused by the $d a f-2(e 1370)$ mutation did not abolish starvation effects on lin3(n378) mutants (Table 1). However, vulval induction in daf-2(e1370) lin-3(n378) double mutants was significantly lower than in the single lin-3(n378) mutant, both in food and starvation conditions. This result is consistent with previous observations that reduced Insulin signaling may lower vulval induction in sensitized backgrounds, such as let-60/Ras (gf) (Battu et al. 2003; Nakdimon et al. 2012). To confirm that TGF- $\beta$ and Insulin signaling do not mediate positive starvation effects on vulval induction, we further examined the role of a central genetic component, the DAF-12 steroid receptor, integrating downstream effects of these two signals (Fielenbach and Antebi 2008) using the Daf-d mutant, daf-12(rh61rh411) (Antebi et al. 2000). Again, as for daf-2 and daf7, this mutant did not abolish positive starvation effects on lin-3(n378) vulval induction (Table 1).

Taken together, these results indicate that Insulin and TGF- $\beta$ pathways, and thus by implication, environmental signal transduction via these pathways, do not play major roles in mediating positive starvation effects on vulval induction. However, we confirm previous observations (Battu et al. 2003; Nakdimon et al. 2012) that reduced DAF-2 Insulin activity may lower vulval induction in sensitized backgrounds, including lin-3(rf) mutants, in both food and starvation conditions. Yet, in our experiments, starvation nevertheless increased vulval induction of daf-2(e1370); lin3(n378) animals, suggesting that the negative starvation effect (Battu et al. 2003; Nakdimon et al. 2012) on vulval induction is significantly weaker than the positive starvation signal. Therefore, while Insulin signaling and sensory perception seem to mediate a mild negative starvation signal (Battu et al. 2003; Nakdimon et al. 2012), we here provide evidence for an additional positive starvation signal acting independently of Insulin and sensory signaling. Moreover, starvation thus appears to induce multiple antagonistic effects on C. elegans vulval induction.

\section{Nutrient-deprivation caused by rsks-1 and pept-1 mutations strongly increase vulval induction in lin-3(rf)}

To test whether observed starvation effects on vulval induction might be mediated by internal perception of the organism's nutritional status, we focused on a conserved key regulator of cell growth and proliferation via nutrient sensing, the LET-363/TOR (target of rapamycin) pathway (Hietakangas and Cohen 2009; Jia et al. 2004). RNAi knockdown of let-363 and rsks-1/S6K (encoding the C. elegans orthologue of mammalian P70S6K, a known TORC1 target) (Laplante and Sabatini 2012), significantly increased vulval induction of $\operatorname{lin}-3(n 378)$ animals in food conditions (Figure 3A). Moreover, RNAi knockdown of pept-1 (encoding an intestinal oligopeptide transporter) (Fei et al. 1998), causing nutrient-deprived animals through amino acid starvation (Meissner et al. 2004), increased vulval induction of $\operatorname{lin}$-3(n378) even more strongly (Figure 3A). 
These RNAi effects thus mimicked starvation effects on lin-3(rf), consistent with the idea that TOR pathway components and targets mediate starvation effects on vulval induction. We further tested whether RNAi knock-down of an additional TOR pathway member daf-15 (RAPTOR) affects vulval induction using the lin-3(n378) mutant. RNAi of daf-15 did not cause any suppression of the Vulvaless phenotype of lin3(n378) (Figure 3A).

To consolidate the above results, we constructed double mutants of lin-3(n378) with rsks-1(ok1255) and pept-1(lg601), respectively. The two double mutants exhibited extremely strong suppression of the lin-3(n378) Vulvaless phenotype (Figures 3B to $3 \mathrm{E})$. The suppression was particularly strong in lin-3(n378); pept-1(lg601) where mean vulval induction approached wild type levels $(2.93 \pm 0.03$ cells induced) (Figure 3E), and $70 \%$ of individuals generated a correct vulval patterning sequence of $2^{\circ}-1^{\circ}-2^{\circ}$ cell fates for P5.p to P7.p compared to $<5 \%$ of lin-3(n378) individuals (Figures 3B and 3C). Similarly, we also observed strong suppression of the Vulvaless phenotype for another lin-3 allele, e1417, when placed in a pept-1(lg601) background [lin-3(e1417); pept1(lg601): $2.13 \pm 0.17$ cells induced, $\mathrm{N}=34$; lin-3(e1417): $0.94 \pm 0.13$ cells induced, $\mathrm{N}=45$; ANOVA, $\left.\mathrm{F}_{1,77}=30.09, \mathrm{P}<0.0001\right]$. Of note, however, neither pept -1 (lg601) or rsks-1(ok1255) single mutants exhibited vulval hyperinduction or other vulval defects ( $N>300 /$ strain). As compromised PEPT-1 activity leads to strongly nutrient-deprived animals, even in a food-rich environment (Meissner et al. 2004; Geillinger et al. 2014), these results are further consistent with our observation that sensory perception of external starvation conditions is not required to increase vulval induction of lin-3(rf) animals (Table 1).

Given that reduced amino acid availability caused by pept-1(lg601) primarily acts through TOR signaling (Meissner et al. 2004; Korta et al. 2012) and that RNAi of rsks-1 similarly mimics starvation effects, we conclude that the observed positive starvation modulation of vulval induction likely involves TOR-S6K signaling.

Although TOR and DAF-2 Insulin signaling may largely act independently of each other, cross-talk between the two pathways has also been reported in some biological processes (Korta et al. 2012; Spanier et al. 2010; Meissner et al. 2004). We therefore tested how pept-1 RNAi affects vulval induction in daf-2(e1370); lin-3(n378) animals: pept-1 RNAi increased vulval induction in a daf-2(e1370); lin-3(n378) double mutants, but to a lesser extent than in lin-3(n378) single mutants (Figure 3F). This result also tightly mimics the observed starvation effects on vulval induction in daf-2(e1370); lin-3(n378) animals (Table 1), corroborating the interpretation that compromised DAF2 signaling causes a slight reduction of vulval induction, as indicated by earlier studies (Battu et al. 2003; Nakdimon et al. 2012). Increased vulval induction caused by pept-1 RNAi or starvation thus largely acts independently of DAF-2/IIR signaling.

In both RNAi and mutant experiments, disruption of PEPT-1 activity caused a stronger increase in lin-3(n378) vulval induction compared to disruption of RSKS-1 activity (Figures $3 \mathrm{~B}$ and $3 \mathrm{C}$ ). Therefore, nutrient deprivation caused by reduced PEPT1 activity did not precisely recapitulate the effects of the $r s k s-1$ mutant, which could indicate that factors other than $r s k s-1$ mediate effects of nutrient deprivation on vulval induction. Consistent with this idea, we found that rsks-1(ok1255); lin-3(n378) double mutants exposed to either pept-1 RNAi or starvation exhibited a slight, but significant increase in vulval induction compared to control animals (Figure 3G). Positive effects of nutrient deprivation on vulval induction may thus involve additional factors other than TOR-S6K signaling. 
Overall, nutrient deprivation - caused by external starvation through absence of food or by internal, physiological starvation due to pept-1 mutation or RNAi - have very similar effects, generating an overall net increase in vulval induction.

pept-1 RNAi increases Ras and Notch pathway activities in vulval precursor cells To address how physiological starvation caused by pept-1 RNAi affects the vulval signaling network, we focused on the main inductive pathway, the Ras pathway. Starvation conditions have previously been shown to increase EGF-Ras-MAPK activity in the $1^{\circ}$ cell, P6.p, and Delta-Notch activity in the $2^{\circ}$ cells, P5.p and P7.p of the wild type strain N2 (Braendle and Félix 2008). We therefore tested whether pept-1 RNAi mimicked these starvation effects using the same fluorescent reporters, egl-17::cfp to quantify Ras pathway activity (Yoo et al. 2004) and lip-1::gfp to quantify Delta-Notch activity (Berset et al. 2001). pept-1 RNAi effects closely mirrored previously quantified starvation effects on report gene activities (Braendle and Félix 2008), with pept-1 RNAi increasing Ras pathway activity in P6.p and increasing Notch pathway activity in P5.p (but not P7.p) (Figures 4A and 4B; Table 2). Consistent with these changes, we also observed that pept-1 RNAi reduced Delta-Notch activity in P6.p while reducing Ras activity in P5.p and P7.p (Figures 4A and 4B; Table 2). pept-1 RNAi thus tightly recapitulated previously observed starvation effects on activities of the two key inductive vulval signaling pathways (Braendle and Félix 2008). Observed changes in pathway activities may be explained by distinct effects on Ras and Notch pathways, or by effects mediated solely by the Ras pathway, given the cross-talk between the two pathways (Yoo et al. 2004).

\section{Starvation sensitivity of vulval induction requires minimal lin-3/egf expression}

To further characterize how physiological starvation interacts with vulval induction to modulate inductive levels, we characterized effects of pept-1 RNAi on several components of the core EGF-Ras-MAPK cascade using various reduction-of-function mutants (Table 3). In addition to lin-3(rf) mutations, pept-1 RNAi suppressed, to different degrees, the Vulvaless phenotypes of let-23(syl), sem-5(n2019) and mpk1(kul) (Table 3). Although these results suggest that nutrient deprivation due to pept-1 RNAi may act downstream of MAPK, interpretation of these effects is limited given that these mutations do not represent null alleles, most of which are homozygous lethals. We therefore used a double mutant let-23(syl); lin-3(n378) that exhibits a fully penetrant Vulvaless phenotype, indicating absence or very low levels of basal Ras activity (Braendle and Félix 2008). Vulval induction of let-23(syl); lin-3(n378) animals treated with pept-1 RNAi remained virtually unaltered compared to controls (Table 3 ), with only $4 / 60$ individuals showing some induced vulval cells (versus 0/45 individuals in food conditions). Very similarly, a recently constructed tissue-specific null allele of lin-3/EGF, mf75 (Barkoulas et al. 2016), did not show any sensitivity to pept-1 RNAi, suggesting that loss of basal EGF-Ras-MAPK activity due to complete lack of LIN-3 abolishes starvation effects. These results indicate that nutrient deprivation via PEPT1 modulates vulval induction upstream of LIN-3 or act in parallel to reinforce expression of downstream target genes of the Ras pathway (as seen with transcriptional reporters).

Given the above results, we next tested whether nutrient deprivation caused by the pept-1 mutation may alter levels of lin-3 expression in the anchor cell. Using single molecule FISH, we therefore compared lin-3 transcript number in wild type, pept1(lg601), lin-3(e1417) and lin-3(e1417); pept-1(lg601) animals (Figure 4C). However, there was no effect of the pept-1 mutation on lin-3 transcript numbers in either a wild 
type genetic background or in a background with low transcriptional level of lin-3. Therefore, increased vulval induction of lin-3(e1417) (Figure 4C) caused by pept-1 mutation is not due to increased transcription of lin-3 in the anchor cell. This result, together with the observation that vulval induction of lin-3(mf75), a genetic background with no expression of lin-3 in the anchor cell, is insensitive to pept-1 RNAi, suggests that starvation effects on vulval induction can only manifest under minimal EGF-RasMAPK activity, corresponding to the situation where one or more lin-3 transcripts are present in the AC. One possible scenario is that the effect of such low doses of lin-3 on vulval induction can then become amplified under nutrient deprivation, e.g. at the level of the LIN-3 protein and its secretion, or at the level or downstream of the EGF receptor (LET-23)

\section{Role of BAR-1 activity in starvation modulation of vulval induction}

In addition to the EGF-Ras-MAPK, starvation effects on vulval induction may also act through additional signaling pathways, in particular, the Wnt pathway. Previous results suggest that partial redundancy between Ras and Wnt pathway in vulval induction may allow for compensatory interplay when one of the two pathways is perturbed due to mutation (Gleason et al. 2002) or exposure to specific environments, such as liquid (Moghal et al. 2003) or starvation (Braendle and Félix 2008). Gleason et al. (2002) observed that Wnt pathway overactivation (by mutation of pry-1) may partly compensate for reduced EGF-Ras-MAPK activity during vulval induction, e.g. in let23(rf) mutants. Braendle \& Félix (2008) further found that the vulval hypoinduction phenotype of the null mutant of bar-1/ $\beta$-Catenin (allele ga80) (Eisenmann et al. 1998) was strongly aggravated under starvation conditions. Moreover, the highly penetrant Vulvaless phenotype of lin-3(n378); bar-1 (ga80) double mutants was not suppressed by starvation, implying that Wnt pathway activity acting via bar-1/ $\beta$-Catenin is required for starvation suppression of $\operatorname{lin}-3(r f)$. However, it remained unclear to what extent starvation compromised induction versus competence of vulval cells in bar1 (ga80) because in the study of Braendle \& Félix (2008) animals were exposed to starvation from the late L1 stage - a stage during which competence of vulval precursor cells is acquired, yet induction does not yet take place. To distinguish these two scenarios, we exposed bar-1 (ga80) animals to starvation in late L1 versus mid-L2 stage and found that mid-L2 starvation did not alter vulval induction whereas late L1 starvation caused strong hypoinduction, primarily due to Pn.p fusion, i.e. loss of competence of vulval precursor cells, including P5.p to P7.p (Figures 5A-E). Late L1 starvation therefore aggravated bar-1 (ga80) fusion defects prior to vulval induction, so that this starvation treatment cannot be used to evaluate starvation effects on vulval inductive levels. In contrast, starvation at a later time point, at mid-L2 - covering the time period during which vulval induction is most sensitive to starvation - slightly, although not significantly, increased vulval induction of bar-1(ga80) animals (Figure 3A). Yet, pept-1 RNAi treatment of bar-1 (ga80) clearly and significantly increased vulval induction (Figure 5F), indicating that such a prolonged starvation stimulus can indeed increase vulval induction in the absence of BAR-1 activity. However, when we exposed lin-3(n378); bar-1 (ga80) double mutants to pept-1 RNAi treatment (Control: $0.00 \pm 0.00$ cells induced, $\mathrm{N}=44$; pept -1 RNAi: $0.03 \pm 0.01$ cells induced, $\mathrm{N}=59$ ), or 48 hours of starvation at the mid-L2 stage (Food: $0.00 \pm 0.00$ cells induced, $N=45$; Starvation: $0.01 \pm 0.01$ cells induced, $\mathrm{N}=47$ ), we observed no starvation suppression of this highly penetrant Vulvaless phenotype. This result suggests, similar to the results obtained for other highly penetrant Vulvaless mutants [e.g. let-23(syl); lin-3(n378) or $\operatorname{lin}-3(m f 75)]$, that a very strong reduction in inductive signals (including contribution 
from BAR-1/Wnt) cannot be rescued by starvation effects. Nevertheless, given that vulval induction of the bar-1 null allele ga 80 increases in response to starvation, we conclude that Wnt pathway/BAR-1 activity is not essential to mediate positive starvation effects on vulval inductive levels.

A previous study has further shown that liquid culture of animals suppressed the Vulvaless phenotype of lin-3(rf) and other EGF-Ras-MAPK mutations through the Wnt pathway (Moghal et al. 2003) (Figure 1C). This environmental effect on vulval induction is based on activation of the heterotrimeric Gaq protein, EGL-30, which acts with muscle-expressed EGL-19 to promote vulval induction downstream or in parallel to LET-60/Ras in a Wnt-dependent manner (Moghal et al. 2003). The liquid effect on vulval induction is abolished when the Wnt pathway is mildly compromised (Moghal et al. 2003), i.e. in a bar-1(mu63) context (Maloof et al. 1999). We therefore asked whether starvation effects on lin-3(rf) could be mediated in the same fashion. However, starvation did not alter vulval induction of lin-3(n378) in a bar-1(mu63) background (Figure 5G). Furthermore, loss of egl-30/G $\alpha q$ (Brundage et al. 1996) did not abolish starvation effects on vulval induction of lin-3(n378) (Figure $5 \mathrm{H})$. Therefore, the positive starvation effects on vulval induction seem to be distinct from previously reported effects of liquid culture promoting vulval induction (Moghal et al. 2003).

\section{Conclusions}

Analysis of starvation sensitivity of $C$. elegans vulval induction using mutant analysis and quantification of pathway activities allowed us to clarify how a specific environmental condition modulates $C$. elegans vulval induction. We present quantitative analyses of starvation effects on vulval induction using lin-3(rf) mutants, demonstrating that starvation exposure of $>19 \mathrm{~h}$ exerts a strong positive effect during the entire period of vulval induction, spanning from mid-L2 to early L3 stages. By testing various candidate mechanisms that could transduce and elicit observed starvation effects, we find that compromised sensory signaling through mutation of daf2 (Insulin) or daf-7 (TGF- $\beta$ ) does not abolish lin-3(rf) starvation suppression. Instead, nutrient-deprived animals induced by mutation of the intestinal peptide transporter pept-1 or by the TOR pathway component $r s k s-1$ (in a food-rich environment) strongly mimicked suppression of lin-3(rf) by external starvation. Moreover, we find that pept1 RNAi is sufficient to increase both EGF-Ras-MAPK and Delta-Notch pathway activities in Pn.p cells. These results provide evidence for a link between nutrient deprivation, TOR-S6K and EGF-Ras-MAPK signaling during C. elegans vulval induction. It remains unclear how nutrient deprivation feeds into genetic pathways regulating vulval development, and we do not know whether this phenomenon represents a specific mechanism to fine-tune levels of vulval induction in response to the environment. In any case, the observed starvation modulation of $C$. elegans vulval induction provides an ideal study system to understand how organismal nutrient status can alter activities of major signaling pathways and penetrance of mutant phenotypes.

A potentially important factor that could partly explain observed starvation effects on vulval induction is prolonged developmental time cause by either starvation exposure and mutation of pept-1 and rsks-1. Applied starvation treatments caused a strong delay in development: animals remained in the L2 stage throughout the 48-hr starvation period (the generation time under normal conditions at $20^{\circ}$ is 3.5 days); and pept-1 and rsks-1 mutants also showed important developmental delays relative to the wild type. In addition, we observed that individuals starved for only short time period ( 2 hours) showed no suppression while longer starvation periods (19-24 hours versus 
48-120 hours) had different effects, with prolonged starvation periods causing increased suppression (Figure 2B). If the vulval signaling network does not operate at steady state, such developmental delays may allow for accumulation of active downstream effectors inducing vulval fates. Previous experiments, using hydroxyurea treatment or anchor cell ablation, supported this hypothesis (Canevascini et al. 2005; Félix 2007; Wang and Sternberg 1999). However, contrary to this hypothesis, slowing developmental speed by lowering growth temperature does not increase vulval induction of lin-3(rf) mutants (Braendle and Félix 2008). Moreover, a mutation in clk1 (a gene necessary for the synthesis of ubiquinone) that causes a 2-fold increase in developmental time (Ewbank et al. 1997; Shibata et al. 2003) does not increase vulval induction in a lin-3(n378) background (data not shown). Thus, a prolonged developmental time alone may not be sufficient to affect vulval induction.

Our results clarify some seemingly contradictory studies reporting both positive (Braendle and Félix 2008; Ferguson and Horvitz 1985) and negative effects (Battu et al. 2003) of starvation on vulval induction. We confirm here that starvation results in a strong and consistent increase in vulval induction, e.g. illustrated by the strong starvation suppression of lin-3(rf) mutations (Figure 2). This positive starvation effect appears to act independently of previously reported transmission of negative starvation effects via specific chemosensory components, Insulin or TGF- $\beta$ signaling (Battu et al. 2003). The study by Battu et al. (2003) used sensitized backgrounds causing vulval hyperinduction (Multivulva phenotype), such as a gain-of-function mutation of let60/Ras, to assess starvation effects: starvation decreased vulval induction in strains with overactivated LET-60 and MPK-1/MAPK signaling, yet had no effect on strains producing excessive LIN-3 or LET-23 (Battu et al. 2003). These results were interpreted to suggest that overstimulation of EGF/EGFR may overcome the negative starvation signal, however, it remained ambiguous at which level of the EGF-RasMAPK cascade the signal may integrate. The negative starvation effects on strains with excessive LET-60 or MPK-1 activation were abolished in chemosensory-defective mutants (osm-5, che-3, sra-13), implying starvation signal transduction via the sensory system (Battu et al. 2003). Moreover, daf-2(rf) suppression of the Multivulva phenotype caused by let-60/Ras $(g f)$ in food conditions was interpreted to suggest mimicking (of observed) starvation effects via reduced Insulin signaling (Battu et al. 2003), but it was not tested how vulval induction of daf-2(rf); let-60(gf) responds to starvation. Our results reporting an overall positive effect of starvation on vulval induction suggest that sensory perception is not required for this effect to occur. Importantly, however, and in line with the results by Battu et al. (2003), we also find that a compromised DAF-2 activity reduces vulval induction in lin-3(rf) to similar extents in both food and starvation conditions, yet without abolishing starvation suppression of lin-3/egf(rf). Together, these different results suggest that starvation has both positive and negative effects on $C$. elegans vulval induction, with a positive starvation signal acting independently of external sensory inputs, likely mediated by nutrient sensing via TOR-S6K (this study), and a sensory-system-mediated negative starvation signal, likely involving DAF-2 signaling (this study; Battu et al. 2003). Despite the presence of two antagonistic starvation signals acting in parallel, there is a strong net positive starvation effect, suggesting that negative starvation effects are significantly weaker.

Our study and several others (Battu et al. 2003; Braendle et al. 2010; Ferguson and Horvitz 1985; Moghal et al. 2003) show that C. elegans vulval cell fate patterning is sensitive to diverse environmental inputs, which may act through distinct mechanisms to affect vulval signaling pathways. While these results show that specific 
developmental processes and underlying genetic pathways are responsive to the environment, several key questions remain: What are the consequences of this environmental sensitivity? How does the observed modulation of vulval signaling impact function and precision of this patterning process? Fundamentally, two opposed hypothetical scenarios can be considered to address these questions. First, environmental sensitivity of vulval development may be inevitable, so that environmental effects represent inherent environmental sensitivity of involved mechanisms. If this scenario holds true, the question is whether such environmental sensitivity translates into deleterious effects, such as patterning defects. In a second scenario, environmental sensitivity may reflect a specific developmental modulation to maintain or to enhance functioning and precision in different environmental conditions. This scenario would imply a vulval developmental system whose environmental flexibility is partly of adaptive origin. It is currently not known which of these scenarios apply to the observed environmental sensitivity of $C$. elegans vulval development. However, a previous study (Braendle and Félix 2008) has quantified functioning and precision of vulval cell fate patterning in different environmental conditions: wild type animals of multiple $C$. elegans isolates showed a very low rate of patterning defects in diverse, harsh environments, including starvation. Although starvation significantly increased levels of vulval induction (Braendle and Félix 2008), vulval patterning errors indicative of such increased inductive levels (e.g. hyperinduction) remained very rare (N2 strain, starvation: 3/1000 versus control: 2/1000 individuals) (Braendle and Félix 2008). Therefore, this type of starvation modulation of vulval signaling pathways does not translate into an increased rate of corresponding errors, indicating that this process tolerates considerable changes in pathway activities. These observations reinforce the notion that the $C$. elegans vulval developmental system is robust to extensive signal fluctuations of involved pathways (Barkoulas et al. 2013; Braendle et al. 2010; Félix and Barkoulas 2012; Hoyos et al. 2011; Milloz et al. 2008). Nevertheless, although current results clearly demonstrate robustness, i.e. tolerance of the vulval developmental system to environmental variation, it remains to be evaluated whether specific environmental modulation of signaling pathways enhances the fidelity and precision of the vulval patterning output. 
Table 1. Effects of starvation treatment on vulval induction level of different mutants of the sensory system. Statistical tests (One-Way-ANOVAs) were performed for each mutant separately testing for the effect of treatment (food vs starvation) on the number of vulval cells induced ${ }^{*} \mathrm{P}<0.05,{ }^{* *} \mathrm{P}<0.01,{ }^{* * *} \mathrm{P}<0.001$, ns: nonsignificant). To facilitate comparison, measurements for lin-3(e1417) and lin-3(n378) are also included (i.e. same data as in Figures $2 \mathrm{C}$ and 2F).

\begin{tabular}{|c|c|c|c|}
\hline Genotype & Treatment & $\begin{array}{l}\text { Induced cells } \\
(\text { Mean } \pm \text { SE) }\end{array}$ & $\mathbf{N}$ \\
\hline $\operatorname{lin}-3(e 1417)$ & $\begin{array}{l}\text { Food } \\
\text { Starvation }\end{array}$ & $\begin{array}{l}0.77 \pm 0.12 \\
1.50 \pm 0.18^{\text {*** }}\end{array}$ & $\begin{array}{l}34 \\
34\end{array}$ \\
\hline $\operatorname{lin}-3(n 378)$ & $\begin{array}{l}\text { Food } \\
\text { Starvation }\end{array}$ & $\begin{array}{l}0.78 \pm 0.12 \\
2.28 \pm 0.20^{\text {*** }}\end{array}$ & $\begin{array}{l}34 \\
34\end{array}$ \\
\hline che-3(e1124); lin-3(n378) & $\begin{array}{l}\text { Food } \\
\text { Starvation }\end{array}$ & $\begin{array}{l}0.94 \pm 0.04 \\
2.53 \pm 0.21^{* * *}\end{array}$ & $\begin{array}{l}60 \\
37\end{array}$ \\
\hline lin-3(n378); osm-5(p813) & $\begin{array}{l}\text { Food } \\
\text { Starvation }\end{array}$ & $\begin{array}{l}0.91 \pm 0.13 \\
2.58 \pm 0.13\end{array}$ & $\begin{array}{l}60 \\
30\end{array}$ \\
\hline $\operatorname{sra}-13(z h 13) ; \operatorname{lin}-3(n 378)$ & $\begin{array}{l}\text { Food } \\
\text { Starvation }\end{array}$ & $\begin{array}{l}0.86 \pm 0.13 \\
2.07 \pm 0.15^{\text {*** }}\end{array}$ & $\begin{array}{l}60 \\
43\end{array}$ \\
\hline$d a f-7(e 1372) ; \operatorname{lin}-3(e 1417)$ & $\begin{array}{l}\text { Food } \\
\text { Starvation }\end{array}$ & $\begin{array}{l}0.26 \pm 0.11 \\
0.92 \pm 0.20^{* *}\end{array}$ & $\begin{array}{l}32 \\
35\end{array}$ \\
\hline daf-2(e1370); lin-3(n378) & $\begin{array}{l}\text { Food } \\
\text { Starvation }\end{array}$ & $\begin{array}{l}0.01 \pm 0.01 \\
1.13 \pm 0.18^{* *}\end{array}$ & $\begin{array}{l}60 \\
60\end{array}$ \\
\hline $\begin{array}{l}\operatorname{lin}-3(n 378) ; \text { daf- } \\
12(\operatorname{rh} 61 \text { rh411) }\end{array}$ & $\begin{array}{l}\text { Food } \\
\text { Starvation }\end{array}$ & $\begin{array}{l}0.34 \pm 0.09 \\
1.77 \pm 0.31^{* * *}\end{array}$ & $\begin{array}{l}60 \\
16\end{array}$ \\
\hline
\end{tabular}


Table 2. Effects of pept-1 RNAi on EGF-Ras-MAPK reporter egl-17::cfp (A) and Delta-Notch reporter lip-1::gfp (B). Results of statistical tests for transcriptional reporter assays (Figure 5). We used an ANOVA testing for the fixed effects of $R N A i$ treatment, Individual (nested in RNAi treatment), Pn.p cell and the interaction between RNAi treatment and Pn.p cell using mean signal intensity as a response variable. Including the effect Individual (RNAi treatment), allowed controlling for the nonindependence of signal intensity in P5.p, P6.p, and P7.p measured in a given individual. Data was log-transformed for analysis.

\section{A) $e g l-17:: c f p$}

\begin{tabular}{llllr} 
Source & DF & Sum of Squares & F Ratio & \multicolumn{1}{l}{ P } \\
\hline RNAi Treatment & 1 & 26.56 & 0.04 & 0.8413 \\
Individual (RNAi Treatment) & 31 & 2792.82 & 0.14 & 1.0000 \\
Pn.p cell & 2 & 538247.46 & 409.63 & $<0.0001$ \\
Pn.p cell x RNAi Treatment & 2 & 20392.73 & 15.52 & $<0.0001$ \\
\hline Error & 62 & 40733.51 & & \\
\hline
\end{tabular}

B) lip-1::gfp

\begin{tabular}{llllc} 
Source & DF & Sum of Squares & F Ratio & \multicolumn{1}{l}{ P } \\
\hline RNAi Treatment & 1 & 456.59 & 0.34 & 0.5607 \\
Individual (RNAi Treatment) & 26 & 24120.94 & 0.70 & 0.8403 \\
Pn.p cell & 2 & 200764.78 & 75.39 & $<0.0001$ \\
Pn.p cell x RNAi Treatment & 2 & 36553.06 & 13.73 & $<0.0001$ \\
\hline Error & 52 & 69237.88 & & \\
\hline
\end{tabular}


Table 3. Effects of pept-1 RNAi treatment on vulval induction level of different mutants of the EGF-Ras-MAPK signaling cascade. Statistical tests (One-WayANOVAs) were performed for each mutant separately testing for the effect of treatment (control versus pept-1 RNAi) on the number of vulval cells induced $\left({ }^{*} \mathrm{P}<0.05,{ }^{* *} \mathrm{P}<\right.$ $0.01,{ }^{* * *} \mathrm{P}<0.001$, ns: non-significant).

\begin{tabular}{|c|c|c|c|}
\hline Genotype & Treatment & $\begin{array}{l}\text { Induced cells } \\
(\text { Mean } \pm \text { SE })\end{array}$ & $\mathbf{N}$ \\
\hline $\operatorname{lin}-3(e 1417)$ & $\begin{array}{l}\text { Control } \\
\text { pept-1 RNAi }\end{array}$ & $\begin{array}{l}0.46 \pm 0.13 \\
1.16 \pm 0.09^{* * *}\end{array}$ & $\begin{array}{l}60 \\
60\end{array}$ \\
\hline $\operatorname{lin}-3(n 378)$ & $\begin{array}{l}\text { Control } \\
\text { pept-1 RNAi }\end{array}$ & $\begin{array}{l}0.27 \pm 0.05 \\
2.26 \pm 0.17^{* * *}\end{array}$ & $\begin{array}{l}60 \\
60\end{array}$ \\
\hline $\operatorname{lin}-3(m f 75)$ & $\begin{array}{l}\text { Control } \\
\text { pept-1 RNAi }\end{array}$ & $\begin{array}{l}0.00 \pm 0.00 \\
0.00 \pm 0.00^{\mathrm{ns}}\end{array}$ & $\begin{array}{l}45 \\
44\end{array}$ \\
\hline let-23(syl) & $\begin{array}{l}\text { Control } \\
\text { pept-1 RNAi }\end{array}$ & $\begin{array}{l}0.12 \pm 0.08 \\
1.36 \pm 0.17^{* * *}\end{array}$ & $\begin{array}{l}48 \\
60\end{array}$ \\
\hline let-23(sy1); lin-3(n378) & $\begin{array}{l}\text { Control } \\
\text { pept-1 RNAi }\end{array}$ & $\begin{array}{l}0.00 \pm 0.00 \\
0.09 \pm 0.06^{\mathrm{ns}}\end{array}$ & $\begin{array}{l}45 \\
60\end{array}$ \\
\hline sem-5(n2019) & $\begin{array}{l}\text { Control } \\
\text { pept-1 RNAi }\end{array}$ & $\begin{array}{l}0.81 \pm 0.15 \\
1.60 \pm 0.12^{\text {*** }}\end{array}$ & $\begin{array}{l}48 \\
55\end{array}$ \\
\hline$m p k-1(k u 1)$ & $\begin{array}{l}\text { Control } \\
\text { pept-1 RNAi }\end{array}$ & $\begin{array}{l}2.69 \pm 0.05 \\
2.99 \pm 0.01\end{array}$ * & $\begin{array}{l}45 \\
45\end{array}$ \\
\hline
\end{tabular}




\section{Acknowledgements}

We thank Marie-Anne Félix and Joao Picao-Osorio for discussion and comments on previous versions of the manuscript. Strains were provided by the lab of Marie-Anne Félix and the CGC, which is funded by NIH Office of Research Infrastructure Programs (P40 OD010440). CB acknowledges financial support by the Fondation ARC pour la Recherche sur le Cancer and the Centre National de la Recherche Scientifique (CNRS). SG was supported by fellowships from the Ministère de l'Enseignement Supérieur et de la Recherche and the Fondation ARC pour la Recherche sur le Cancer. AMVV was supported by the French government through ANR10-LABX-54 MEMOLIFE.

\section{References}

Antebi, A., W.H. Yeh, D. Tait, E.M. Hedgecock, and D.L. Riddle, 2000 daf-12 encodes a nuclear receptor that regulates the dauer diapause and developmental age in C. elegans. Genes Dev 14 (12):1512-1527.

Barkoulas, M., J.S. Van Zon, J. Milloz, A. Van Oudenaarden, and M.-A. Félix, 2013 Robustness and epistasis in the C. elegans vulval signaling network revealed by pathway dosage modulation. Developmental Cell 24 (1):64-75.

Barkoulas, M., A.M. Vargas Velazquez, A.E. Peluffo, and M.A. Felix, 2016 Evolution of New cis-Regulatory Motifs Required for Cell-Specific Gene Expression in Caenorhabditis. PLoS Genet 12 (9):e1006278.

Battu, G., E.F. Hoier, and A. Hajnal, 2003 The C. elegans G-protein-coupled receptor SRA-13 inhibits RAS/MAPK signalling during olfaction and vulval development. Development 130:2657-2577.

Berset, T., E.F. Hoier, G. Battu, S. Canevascini, and A. Hajnal, 2001 Notch inhibition of RAS signaling through MAP kinase phosphatase LIP-1 during C. elegans vulval development. Science 291:1055-1058.

Braendle, C., C.F. Baer, and M.-A. Félix, 2010 Bias and evolution of the mutationally accessible phenotypic space in a developmental system. PLoS Genetics 6 (3): 1000877.

Braendle, C., and M.-A. Félix, 2008 Plasticity and errors of a robust developmental system in different environments. Developmental Cell 15 (5):714-724.

Braendle, C., and M.A. Felix, 2009 The other side of phenotypic plasticity: a developmental system that generates an invariant phenotype despite environmental variation. J Biosci 34 (4):543-551.

Braendle, C., J. Milloz, and M.-A. Félix, 2008 Mechanisms and evolution of environmental responses in Caenorhabditis elegans. Current topics in developmental biology 80:171-207.

Brenner, S., 1974 The genetics of Caenorhabditis elegans. Genetics 77:71-94.

Bruinsma, J.J., T. Jirakulaporn, A.J. Muslin, and K. Kornfeld, 2002 Zinc ions and cation diffusion facilitator proteins regulate Ras-mediated signaling. Dev Cell $2(5): 567-578$.

Brundage, L., L. Avery, A. Katz, U.J. Kim, J.E. Mendel et al., 1996 Mutations in a C. elegans Gqalpha gene disrupt movement, egg laying, and viability. Neuron 16 (5):999-1009.

Canevascini, S., M. Marti, E. Fröhli, and A. Hajnal, 2005 The Caenorhabditis elegans homologue of the proto-oncogene ect-2 positively regulates RAS signalling during vulval development. EMBO Reports 6:1169-1175. 
Dalfo, D., D. Michaelson, and E.J. Hubbard, 2012 Sensory regulation of the $C$. elegans germline through TGF-beta-dependent signaling in the niche. Curr Biol 22 (8):712-719.

Eisenmann, D.M., J.N. Maloof, J.S. Simske, C. Kenyon, and S.K. Kim, 1998 The bcatenin homolog BAR-1 and LET-60 Ras coordinately regulate the Hox gene lin-39 during Caenorhabditis elegans vulval development. Development 125:3667-3680.

Ewbank, J.J., T.M. Barnes, B. Lakowski, M. Lussier, H. Bussey et al., 1997 Structural and functional conservation of the Caenorhabditis elegans timing gene clk-1. Science 275 (5302):980-983.

Fei, Y.J., T. Fujita, D.F. Lapp, V. Ganapathy, and F.H. Leibach, 1998 Two oligopeptide transporters from Caenorhabditis elegans: molecular cloning and functional expression. Biochem J 332 ( Pt 2):565-572.

Félix, M.-A., 2007 Cryptic quantitative evolution of the vulva intercellular signaling network in Caenorhabditis. Current Biology 17:103-114.

Félix, M.-A., 2012 Caenorhabditis elegans vulval cell fate patterning. Physical Biology 9 (4):045001.

Félix, M.-A., and M. Barkoulas, 2012 Robustness and flexibility in nematode vulva development. Trends in genetics : TIG.

Félix, M.A., and M. Barkoulas, 2015 Pervasive robustness in biological systems. Nat Rev Genet 16 (8):483-496.

Ferguson, E., and H.R. Horvitz, 1985 Identification and characterization of 22 genes that affect the vulval cell lineages of Caenorhabditis elegans. Genetics 110:17-72.

Fielenbach, N., and A. Antebi, 2008 C. elegans dauer formation and the molecular basis of plasticity. Genes Dev 22 (16):2149-2165.

Flatt, T., G.V. Amdam, T.B. Kirkwood, and S.W. Omholt, 2013 Life-history evolution and the polyphenic regulation of somatic maintenance and survival. Q Rev Biol 88 (3):185-218.

Geillinger, K.E., K. Kuhlmann, M. Eisenacher, P. Giesbertz, H.E. Meyer et al., 2014 Intestinal amino acid availability via PEPT- 1 affects TORC1/2 signaling and the unfolded protein response. J Proteome Res 13 (8):3685-3692.

Gleason, J.E., H.C. Korswagen, and D.M. Eisenmann, 2002 Activation of Wnt signaling bypasses the requirement for RTK/Ras signaling during C. elegans vulval induction. Genes Dev. 16:1281-1290.

Greenwald, I.S., P.W. Sternberg, and H.R. Horvitz, 1983 The lin-12 locus specifies cell fates in Caenorhabditis elegans. Cell 34:435-444.

Grimbert, S., and C. Braendle, 2014 Cryptic genetic variation uncovers evolution of environmentally sensitive parameters in Caenorhabditis vulval development. Evol Dev 16 (5):278-291.

Grimbert, S., K. Tietze, M. Barkoulas, P.W. Sternberg, M.A. Felix et al., 2016 Anchor cell signaling and vulval precursor cell positioning establish a reproducible spatial context during C. elegans vulval induction. Dev Biol 416 (1):123-135.

Hietakangas, V., and S.M. Cohen, 2009 Regulation of tissue growth through nutrient sensing. Annu Rev Genet 43:389-410.

Hill, R., and P. Sternberg, 1992 The gene lin-3 encodes an inductive signal for vulval development. Nature 358 (6386):470-476. 
Hoyos, E., K. Kim, J. Milloz, M. Barkoulas, J.-B. Pénigault et al., 2011 Quantitative Variation in Autocrine Signaling and Pathway Crosstalk in the Caenorhabditis Vulval Network. Current Biology:1-12.

Jia, K., D. Chen, and D.L. Riddle, 2004 The TOR pathway interacts with the insulin signaling pathway to regulate C. elegans larval development, metabolism and life span. Development 131 (16):3897-3906.

Kamath, R.S., A.G. Fraser, Y. Dong, G. Poulin, R. Durbin et al., 2003 Systematic functional analysis of the Caenorhabditis elegans genome using RNAi. Nature 421 (6920):231-237.

Korta, D.Z., S. Tuck, and E.J. Hubbard, 2012 S6K links cell fate, cell cycle and nutrient response in C. elegans germline stem/progenitor cells. Development 139 (5):859-870.

Laplante, M., and D.M. Sabatini, 2012 mTOR signaling in growth control and disease. Cell 149 (2):274-293.

Maloof, J.N., J. Whangbo, J.M. Harris, G.D. Jongeward, and C. Kenyon, 1999 A Wnt signaling pathway controls hox gene expression and neuroblast migration in C. elegans. Development 126 (1):37-49.

Meissner, B., M. Boll, H. Daniel, and R. Baumeister, 2004 Deletion of the intestinal peptide transporter affects insulin and TOR signaling in Caenorhabditis elegans. J Biol Chem 279 (35):36739-36745.

Michaelson, D., D.Z. Korta, Y. Capua, and E.J. Hubbard, 2010 Insulin signaling promotes germline proliferation in C. elegans. Development 137 (4):671-680.

Milloz, J., F. Duveau, I. Nuez, and M.-A. Félix, 2008 Intraspecific evolution of the intercellular signaling network underlying a robust developmental system. Genes \&amp; Development 22 (21):3064-3075.

Moghal, N., L.R. Garcia, L.A. Khan, K. Iwasaki, and P.W. Sternberg, 2003 Modulation of EGF receptor-mediated vulva development by the heterotrimeric G-protein Gaq and excitable cells in C. elegans. Development 130:4553-4566.

Nakdimon, I., M. Walser, E. Frohli, and A. Hajnal, 2012 PTEN negatively regulates MAPK signaling during Caenorhabditis elegans vulval development. PLoS Genet 8 (8):e1002881.

Paaby, A.B., and M.V. Rockman, 2014 Cryptic genetic variation: evolution's hidden substrate. Nat Rev Genet 15 (4):247-258.

Penigault, J.B., and M.A. Félix, 2011 High sensitivity of C. elegans vulval precursor cells to the dose of posterior Wnts. Dev Biol 357 (2):428-438.

Rual, J.F., J. Ceron, J. Koreth, T. Hao, A.S. Nicot et al., 2004 Toward improving Caenorhabditis elegans phenome mapping with an ORFeome-based RNAi library. Genome Res 14 (10B):2162-2168.

Salser, S.J., C.M. Loer, and C. Kenyon, 1993 Multiple HOM-C gene interactions specify cell fates in the nematode central nervous system. Genes Dev 7 (9):1714-1724.

Seetharaman, A., P. Cumbo, N. Bojanala, and B.P. Gupta, 2010 Conserved mechanism of Wnt signaling function in the specification of vulval precursor fates in C. elegans and C. briggsae. Dev Biol 346 (1):128-139.

Shibata, Y., R. Branicky, I.O. Landaverde, and S. Hekimi, 2003 Redox regulation of germline and vulval development in Caenorhabditis elegans. Science 302 (5651):1779-1782.

Sokal, R.R., and F.J. Rohlf, 1981 Biometry, 3nd edition. New York: W.H. Freeman and Cie. 
Spanier, B., I. Rubio-Aliaga, H. Hu, and H. Daniel, 2010 Altered signaling from germline to intestine pushes daf-2;pept-1 C. elegans into extreme longevity. Aging Cell:no-no.

Sternberg, P.W., 2005 Vulval development in WormBook, edited by T.C.e.R. Community.

Sternberg, P.W., and H.R. Horvitz, 1986 Pattern formation during vulval development in Caenorhabditis elegans. Cell 44:761-772.

True, J.R., and E.S. Haag, 2001 Developmental system drift and flexibility in evolutionary trajectories. Evol. Dev. 3:109-119.

Wang, M., and P.W. Sternberg, 1999 Competence and commitment of Caenorhabditis elegans vulval precursor cells. Dev. Biol. 212:12-24.

Wood, W.B., 1988 The nematode Caenorhabditis elegans. Cold Spring Harbor, New York: Cold Spring Harbor Laboratory.

Yoder, J.H., H. Chong, K.L. Guan, and M. Han, 2004 Modulation of KSR activity in Caenorhabditis elegans by Zn ions, PAR-1 kinase and PP2A phosphatase. EMBO J 23 (1):111-119.

Yoo, A.S., C. Bais, and I. Greenwald, 2004 Crosstalk between the EGFR and LIN12/Notch pathways in C. elegans vulval development. Science 303:663-666.

Zand, T.P., D.J. Reiner, and C.J. Der, 2011 Ras effector switching promotes divergent cell fates in C. elegans vulval patterning. Dev Cell 20 (1):84-96. 


\section{Figure legends}

Figure 1. Progression and environmental sensitivity of $C$. elegans vulval cell fate patterning. (A) A subset of six Pn.p cells (P3.p to P8.p) acquires competence to form vulval tissue through the expression of LIN-39; however, usually only P5.p, P6.p and P7.p adopt vulval cell fates in a stereotypical $2^{\circ}-1^{\circ}-2^{\circ}$. The induction of vulval cell fates is generated by the cross-talk of EGF-Ras-MAPK and Delta-Notch signaling pathways. Distinct vulval cell divisions according to cell fate occur during the L3 stage and generate 22 vulval cells. (B) The anchor cell (AC) releases the LIN-3 inductive signal. LIN-3 acts as a morphogen, with P6.p receiving the highest level of LIN-3 causing it to adopt a $1^{\circ}$ cell fate (blue). The expression of the Delta ligands in P6.p activates the Delta-Notch pathway in its neighbours, P5.p and P7.p. This activation causes them to adopt a $2^{\circ}$ fate (red) and represses the primary fate. A switch from the canonical LET60/Ras-LIN-45/Raf pathway to a LET-60/Ras-RGL-1-RAL-1 signaling pathway can also promote the $2^{\circ}$ cell fate in P5.p and P7.p (not detailed). The competent cells, P4.p and P8.p, adopt a non-vulval $3^{\circ}$ fate (not represented), while P3.p may either adopt a $3^{\circ}$ fate or a $4^{\circ}$ fate, also referred to as F(used) fate. (C) Schematic representation of previously reported environmental and physiological inputs affecting C. elegans vulval induction (for details, see introduction). Colour coding: Braendle \& Félix (2008): pink, Bruinsma et al. (2002): brown, Battu et al. (2003): red, Moghal et al. (2003): blue, Nakdimon et al. (2012): orange, Grimbert \& Braendle (2014): purple.

Figure 2. Quantifying starvation effects on vulval induction in lin-3/egf(rf) mutants. (A) Differences in vulval induction of lin-3(n378) animals exposed to starvation (48 hours) at different developmental stages. Starvation suppression of lin3(n378) differed significantly between exposed developmental stages (ANOVA, $\mathrm{F}_{5,239}$ $=14.02, \mathrm{P}<0.0001$ ), with strongest effects observed from mid L2 to early L3 stages. (B) Effects of starvation duration on vulval induction of lin-3(n378). Animals (agesynchronized by egg-laying windows) were exposed to starvation at the mid L2 stage for different time periods except for the first treatment where animals were starved in liquid (M9 buffer) for 12 hours directly after hatching ( $\mathrm{N}=80$ per treatment). Starvation duration significantly affected levels of vulval induction (ANOVA, $\mathrm{F}_{5,239}=14.02, \mathrm{P}<$ $0.0001)$ with strongest suppression of $\operatorname{lin}-3(n 378)$ at 48 to 120 hours of starvation. (C) Starvation significantly increased vulval induction of lin-3(n378) animals relative to control food conditions (ANOVA, $\mathrm{F}_{1,66}=33.75, \mathrm{P}<0.0001$ ). (D, E) Individual lin3(n378) fate patterns of P4.p to P8.p in (D) food versus (E) starvation conditions. (F) Starvation significantly increased vulval induction of lin-3(e1417) animals relative to control food conditions (ANOVA, $\left.F_{1,66}=9.97, P=0.0024\right)$. $(\mathbf{G}, \mathbf{H})$ Individual lin3(e1417) fate patterns of P4.p to P8.p in (G) food versus $(\mathbf{H})$ starvation conditions.

Values represented by bars $(\mathbf{A}, \mathbf{B}, \mathbf{C}, \mathbf{F})$ indicate the mean number of induced vulval cells, also referred to as the vulval index (WT=3 cells induced). Values with different letters indicate significant differences (ANOVA, followed by Tukey's HSD) $\left({ }^{*} \mathrm{P}<0.05,{ }^{* *} \mathrm{P}<0.01,{ }^{* * *} \mathrm{P}<0.001\right.$, ns: non-significant $)$. Numbers displayed in bars represent the number of individuals scored; error bars indicate \pm 1 SEM.

Vulval cell fate patterns of P4.p to P8.p (D,E,G,H) were, whenever feasible, separately inferred for Pn.pa and Pn.pp in cases of half-induced fates. Each line represents the vulval pattern of a single individual, and individuals are ordered from highest to lowest index of vulval induction. Colour coding of vulval cell fates $\left(1^{\circ}\right.$ : blue, $2^{\circ}:$ red) and non-vulval cell fates $\left(3^{\circ}:\right.$ yellow). Induced vulval cells that could not be clearly assigned to either a $1^{\circ}$ or $2^{\circ}$ fate are shown in purple. 
Figure 3. Nutrient-deprivation caused by rsks-1 and pept-1 mutations strongly increases vulval induction in lin-3/egf(rf). (A) Effects of let-363, daf-15, rsks-1 and pept-1 RNAi versus control RNAi treatment (empty vector strain, E. coli HT115) of lin-3(n378) animals (food conditions). RNAi knock-down of let-363, rsks-1 and pept1 (but not daf-15) significantly increased vulval induction (ANOVA, all $\mathrm{P}<0.01$ ). (B) rsks-1(ok1255) strongly increased vulval induction of lin-3(n378) animals (ANOVA, $\left.\mathrm{F}_{1,115}=48.42, \mathrm{P}<0.0001\right)$. (C) pept-1(lg601) strongly increased vulval induction of lin-3(n378) animals (ANOVA, $\left.\mathrm{F}_{1,118}=335.58, \mathrm{P}<0.0001\right)$. (D, E) Individual fate patterns of P4.p to P8.p in (D) lin-3(n378) versus (E) lin-3(n378); pept-1(lg601) individuals. Vulval cell fate patterns of P4.p to P8.p (D,E) were, whenever feasible, separately inferred for Pn.pa and Pn.pp in cases of half-induced fates. Each line represents the vulval pattern of a single individual, and individuals are ordered from highest to lowest index of vulval induction. Colour coding of vulval cell fates $\left(1^{\circ}:\right.$ blue, $2^{\circ}$ : red) and non-vulval cell fates $\left(3^{\circ}:\right.$ yellow). Induced vulval cells that could not be clearly assigned to either a $1^{\circ}$ or $2^{\circ}$ fate are coded in purple. (F) Effects of pept-1 RNAi versus control RNAi (empty vector strain, E. coli HT115) in daf-2(e1370); lin-3(n378) (food conditions). RNAi knock-down of pept-1 significantly increased vulval induction (ANOVA, $F_{1,73}=133.57, \mathrm{P}<0.0001$ ). (G) 1) Starvation significantly increased vulval induction of rsks-1(ok1255); lin-3(n378) animals relative to control food conditions (ANOVA, $\left.\mathrm{F}_{1,102}=11.62, \mathrm{P}=0.0009\right)$ 2) Effects of pept 1 RNAi versus control RNAi (empty vector strain, E. coli HT115) in rsks-1(ok1255); lin-3(n378) (food conditions). RNAi knock-down of pept- 1 significantly increased vulval induction (ANOVA, $\mathrm{F}_{1,79}=$ 25.93, $\mathrm{P}<0.0001)$.

Figure 4. Reduced activity of PEPT-1 increases Ras and Notch pathway activities but does not alter lin-3 transcript levels. (A,B) Effects of pept-1 RNAi on the level of the transcriptional reporters egl-17::cfp (EGF-Ras-MAPK activity) (Yoo et al. 2004) and lip-1::gfp (Delta-Notch activity) (Berset et al. 2001) quantified in lethargus L2/L3 and early L3 stages. (A) Mean signal (pixel) intensity of the EGF-Ras-MAPK pathway reporter, egl-17::cfp in P5.p to P7.p. Values indicate Least Square Means for the interaction cell $\mathrm{x}$ RNAi treatment $\left(\mathrm{F}_{2,62}=15.52, \mathrm{P}<0.0001\right)$ (Table $\left.2 \mathrm{~A}\right)$. (B) Mean signal (pixel) intensity of the Delta-Notch pathway reporter, lip-1::gfp in P5.p to P7.p. Values indicate Least Square Means for the interaction cell $\mathrm{x}$ RNAi treatment $\left(\mathrm{F}_{2,52}=\right.$ 13.73, $\mathrm{P}<0.0001$ ) (Table 2B). For complete statistical analysis and results, see Table 2. Values with different letters indicate significant differences (Tukey's HSD). Numbers displayed in bars represent the number of individuals scored; error bars indicate \pm 1 SEM. (C) Boxplot showing lin-3 mRNA levels in different genetic backgrounds [wild type, pept-1(lg601), lin-3(e1417) and lin-3(e1417); pept-1(lg601)] is displayed. The pept-1 mutation does not alter the lin-3 expression level (mean $=$ $24.38 \pm 0.67 ; \mathrm{n}=34$ ) compared to the wild type (mean $=25.36 \pm 0.35 ; \mathrm{n}=22$ ) (ANOVA, $\left.\mathrm{F}_{1,54}=0.49, \mathrm{P}=0.49\right)$. As expected, there is little expression in lin-3(e1417) mutant animals (Mean: $0.77 \pm 0.35, \mathrm{n}=31$ ) but this low expression is not rescued in lin-3; pept1 double mutant animals (Mean: $0.36 \pm 0.17 ; \mathrm{n}=22)\left(\right.$ ANOVA, $\left.\mathrm{F}_{1,751}=0.46, \mathrm{P}=0.50\right)$.

Figure 5. Role of BAR-1 activity in starvation modulation of vulval induction. (A) Differences in vulval induction of bar-1(ga80) animals exposed to 48 hours of starvation in late L1 versus mid L2 (and versus control food conditions). Starvation at the late L1 stage, but not at the mid L2 stage, significantly reduced vulval induction of bar-1 (ga80) animals (ANOVA, $\left.\mathrm{F}_{2,121}=50.27, \mathrm{P}<0.0001\right)$. (B-D) Individual bar- 
1 (ga80) fate patterns of P3.p to P8.p in (B) food versus starvation conditions: late L1 (C) or mid L2 starvation (D). Vulval cell fate patterns of P3.p to P8.p (B-D) were, whenever feasible, separately inferred for Pn.pa and Pn.pp in cases of half-induced fates. Each line represents the vulval pattern of a single individual, and individuals are ordered from highest to lowest index of vulval induction. Colour coding of vulval cell fates $\left(1^{\circ}\right.$ : blue, $2^{\circ}$ : red) and non-vulval cell fates $\left(3^{\circ}\right.$ : yellow, $4^{\circ}$ : grey). Induced vulval cells that could not be clearly assigned to either a $1^{\circ}$ or $2^{\circ}$ fate are coded in purple; noninduced cells that could not be clearly assigned a $3^{\circ}$ or $^{\circ}$ fate are coded in white. (E) Proportion of Pn.p cells with a $4^{\circ}$ (Fused) fate in control, starvation-exposed late L1, starvation-exposed mid L2 bar-1 (ga80) individuals (from the same experiment as shown in (A)). The proportions of $4^{\circ}$ fates for each of P5.p to P7.p cell were higher after L1 starvation (P5.p: 37\%, P6.p: 23\%, P7.p: 44\%) compared to control (P5.p: 2\%, P6.p and P7.p: 0\%) and L2 starvation (P5.p: 12\%, P6.p: 0\%, P7.p: 3\%). (F) pept-1 RNAi increases vulval induction of bar-1 (ga80) animals (ANOVA, $\mathrm{F}_{1,81}=14.47, \mathrm{P}=$ 0.0003). (G) Starvation increased vulval induction of lin-3(n378); bar-1 (mu63) relative to control food conditions (ANOVA, $\mathrm{F}_{1,98}=18.84, \mathrm{P}<0.0001$ ). (H) Starvation increased vulval induction of egl-30(ad805); $\operatorname{lin} 3$ (n378) relative to control food conditions (ANOVA, $\mathrm{F}_{1,59}=7.28, \mathrm{P}=0.0091$ ). 


\section{Figure 1}

A

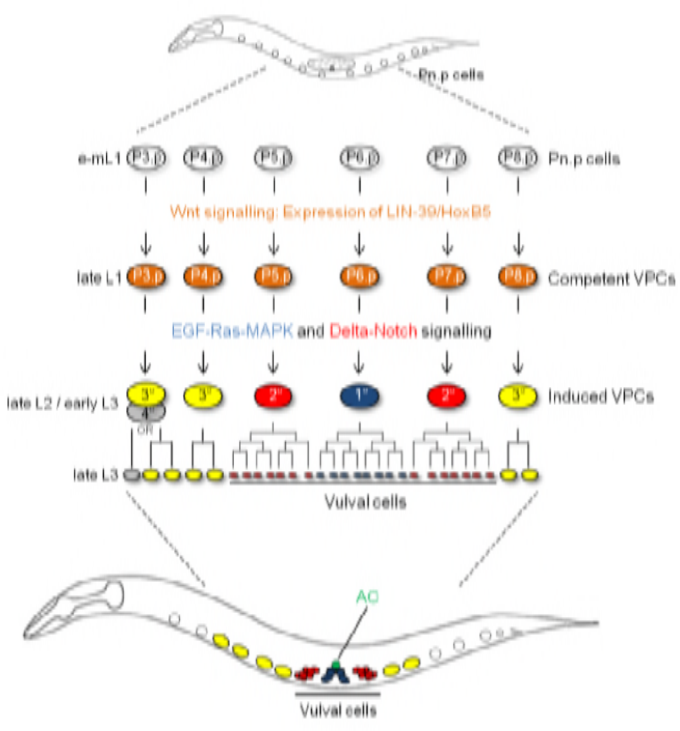

B

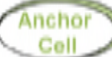

\section{LIN-3/EGF}

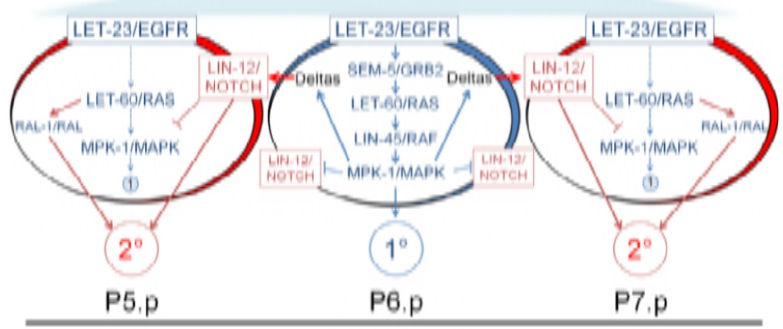

Vulval fates

C

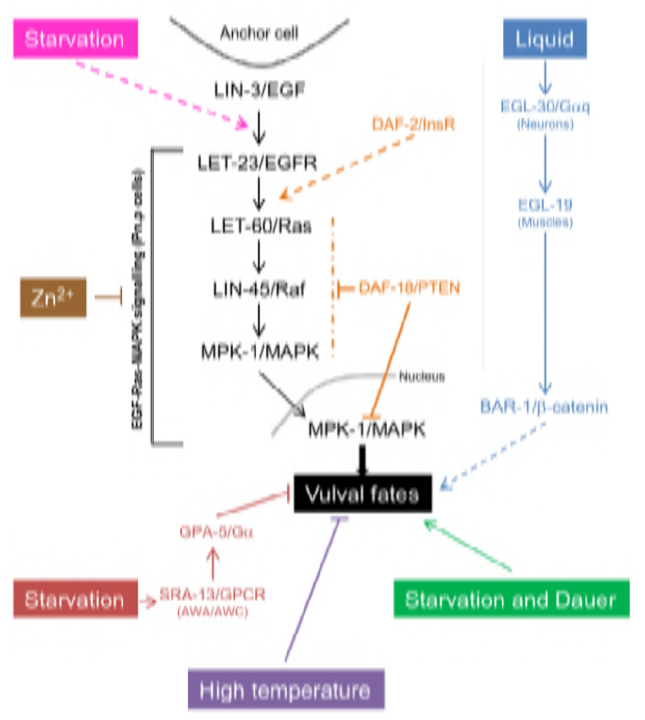


Figure 2
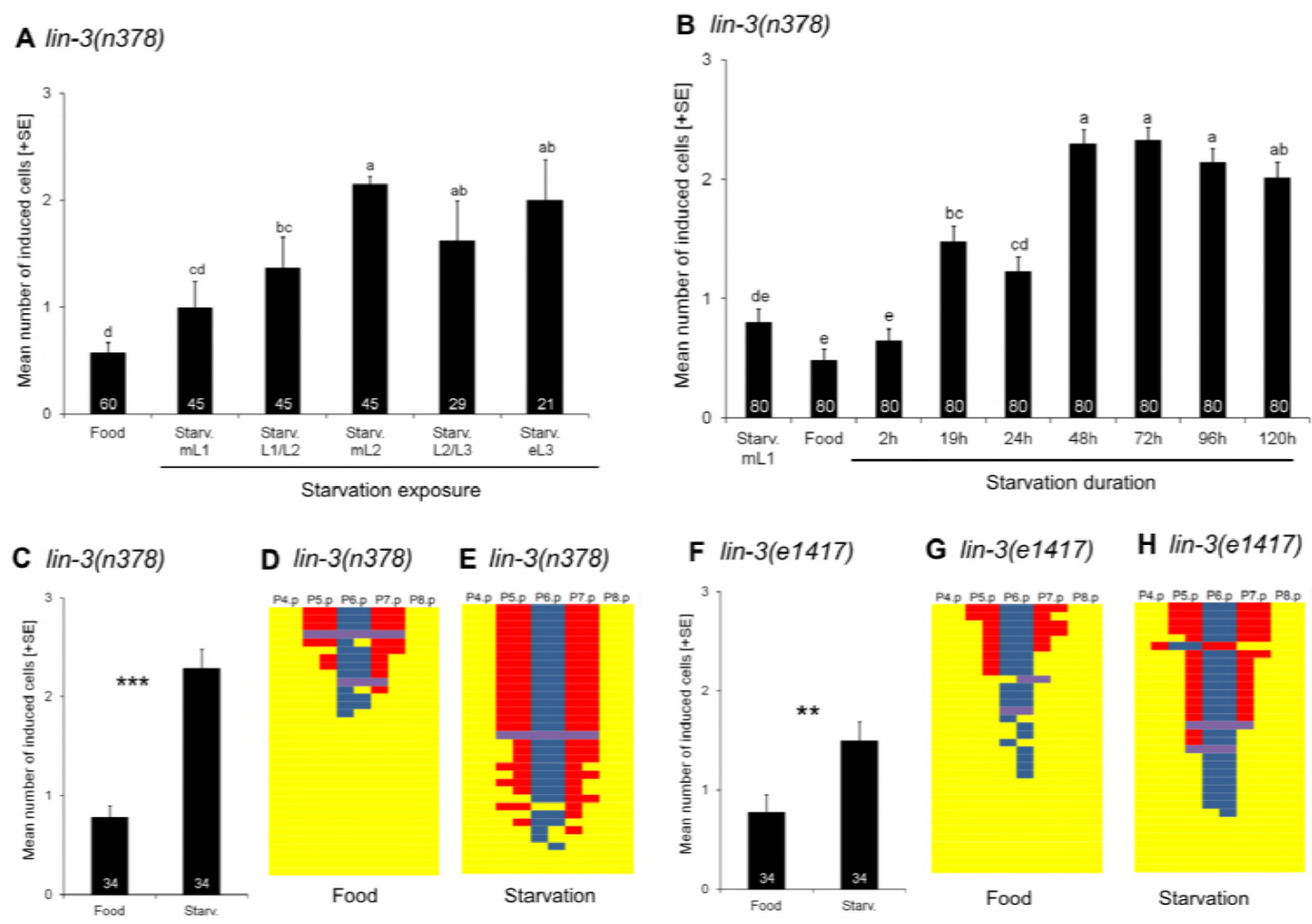

H lin-3(e1417)

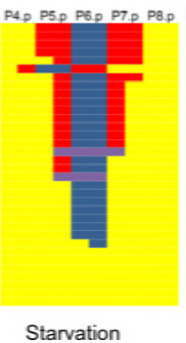




\section{Figure 3}
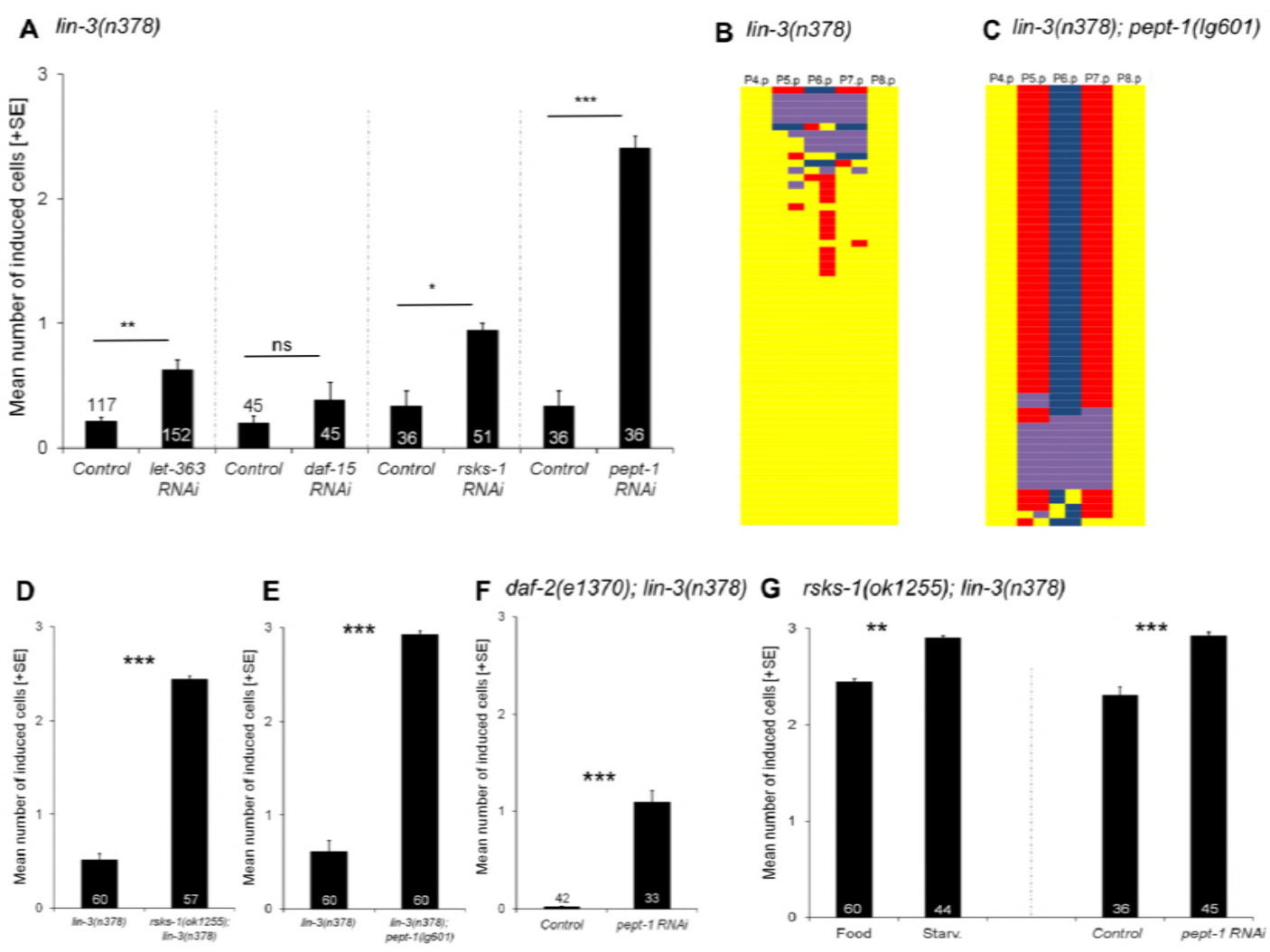


\section{Figure 4}

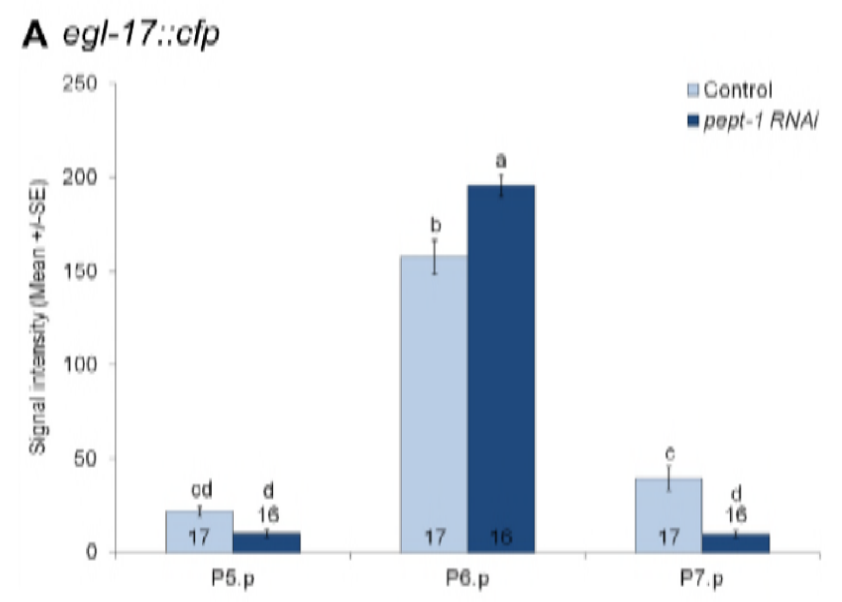

\section{B lip-1:igfp}

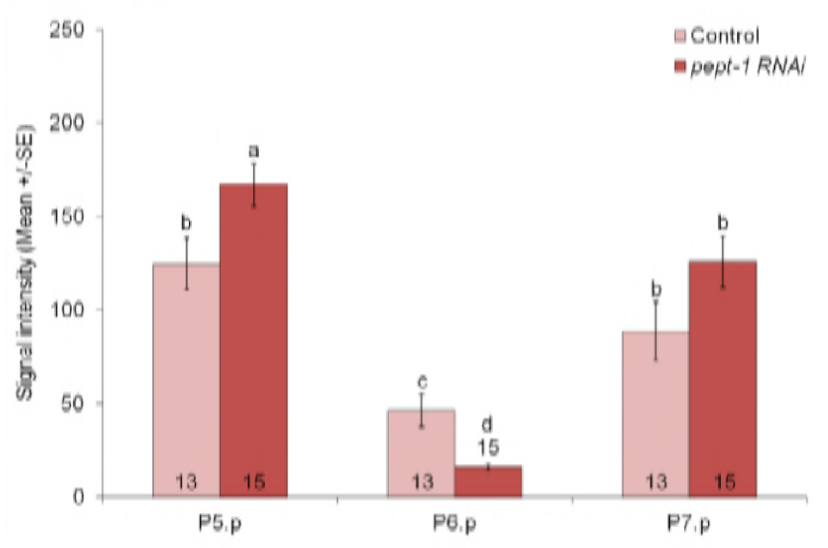

C lin-3 expression levels

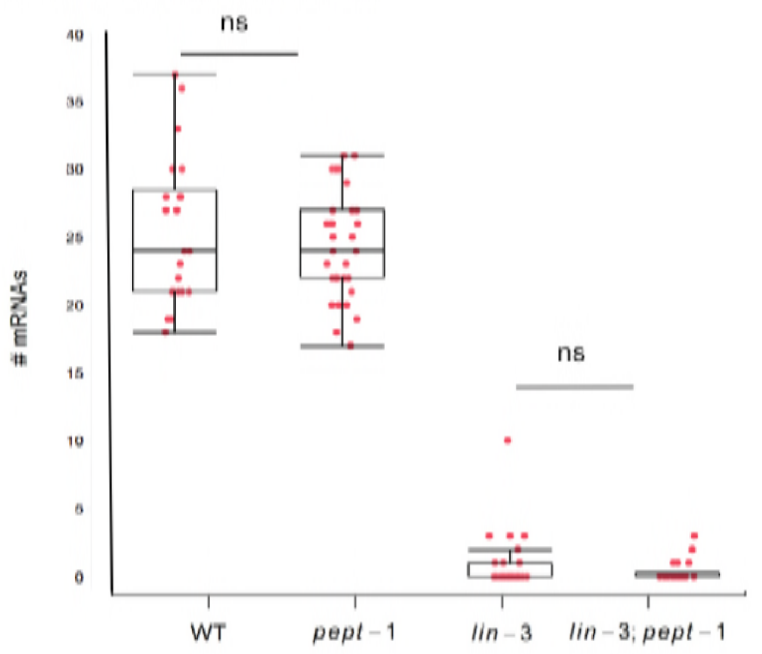




\section{Figure 5}
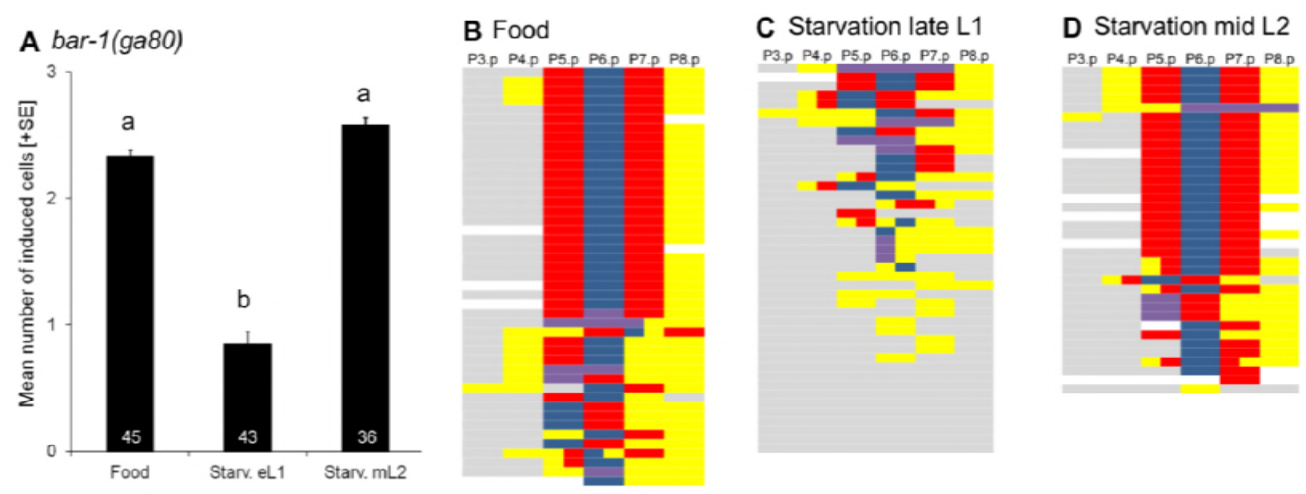

E bar-1(ga80)

F bar-1(ga80)

G lin-3(n378); bar-1(mu63)

H egl-30(ad805); lin-3(n378)
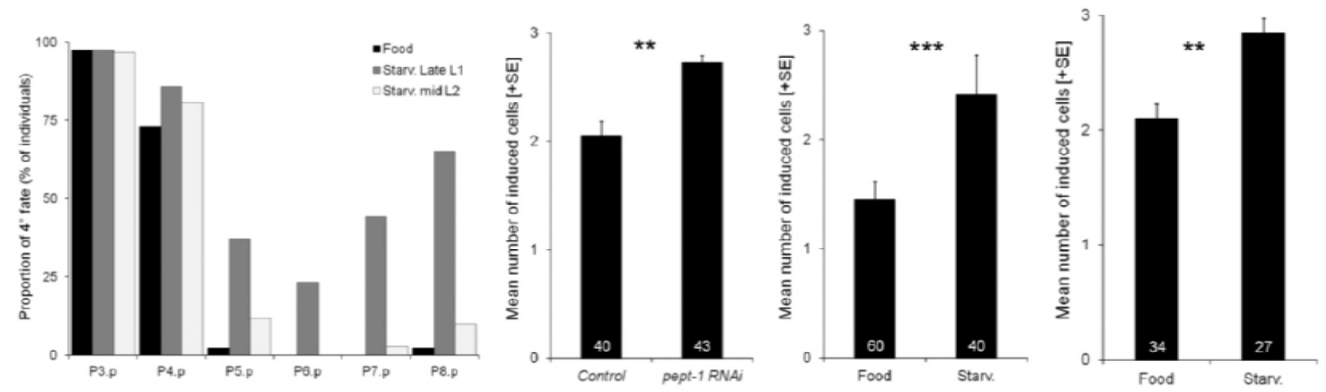